\title{
Uncertainty in the Generic Versus Brand Name Prescription Decision
}

\author{
Jörgen Hellström* and Niklas Rudholm* \\ Department of Economics, Umeå University \\ SE-901 87, Umeå, Sweden \\ email: niklas.rudholm@econ.umu.se \\ tel: +46-90-7869940 \\ fax: $+46-90-772302$
}

January 16, 2003

\begin{abstract}
This paper analyzes the impact of uncertainty concerning product quality of generic drugs on the substitution behavior of prescribing physicians. It is shown that uncertainty about the generic drug quality gives the physician a value of waiting for more information before switching to the generic version. In addition, it is shown that reducing the approval requirements for generic drugs, thereby increasing uncertainty about quality, may discourage physicians from prescribing such drugs. A small empirical study supports the theoretical findings and indicate that uncertainty about the quality of generic drugs do affect physician prescription behavior. (JEL L65, I11)
\end{abstract}

\footnotetext{
${ }^{*}$ Financial support from the Jan Wallander and Tom Hedelius foundation is greatly acknowledged.
} 


\section{Introduction}

Policies designed to improve the efficiency of pharmaceutical markets have been implemented both in the US and in Europe. The (US) Drug Competition and Patent Term Restoration Act of 1984 (also known as the WaxmanHatch Act) had two objectives: to restore the effective patent terms, which had eroded substantially over the years due to more complex and timeconsuming approval procedures (Hartley et al, 1986, and Andersson and Hertzman, 1993) and to increase generic competition once patents expire. In order to increase generic competition, approval procedures for generic drugs were changed considerably. Instead of having to redo all clinical trials themselves, a generic product can now be approved by showing that it is bioeqivalent to the brand name product. The testing procedures required to do this are less costly than the original safety and efficacy tests that the brand name manufacturer has to conduct. Similar changes in approval procedures have been made in Europe. In Sweden, for example, an applicant for a license to sell a generic drug does not have to produce pharmacological and toxicological studies if he can prove that the new pharmaceutical is equal to another pharmaceutical product that has been approved for sale in Sweden or another country within the European Union and is, at present, sold in Sweden. ${ }^{1}$ As such, both US and European governments have changed the approval procedures for generic drugs in order to increase generic entry and competition in the pharmaceuticals market.

However, for these measures to be effective, the prescribing physicians must be willing to switch from the brand name to the generic version of the drug. Fridman et al (1987) reports that only half of 245 surveyed physicians believed generic drugs to be as reliable as brand name drugs. Consistent with this result, a relatively low fraction of physicians report to prescribe generics often, except in the case of antibiotics. Although 60 percent of physicians often comply with patients, should patients request a generic prescription, the impact of this is not large since patients do not often make such requests.

One factor that affects the confidence in the quality of generic drugs concerns the quality control done by approving authorities such as the US Food and Drug Administration (FDA). In 1989 a scandal concerning bribed employees at the FDA emerged (see e.g., Scott-Morton, 1997, 1999). The

\footnotetext{
${ }^{1}$ These procedures described follow the Medical Products Agency rule 1993:14. The preceding rules for approval can be found in the National Board of Health and Welfare Code of Statutes 1980:90.
} 
pharmaceutical company Mylan filed a complaint stating that its competitors applications received faster review than their applications. Investigations into these accusations showed that several generic firms had been bribing FDA reviewers in order to speed up approval. ${ }^{2}$ Gupta (1996) reports that the increased uncertainty about the quality of generic products following the scandal affected the confidence of pharmacists, physicians and consumers using such drugs. In addition, Gupta reports that a majority of pharmacists switched to using more brand name drugs in their personal consumption of pharmaceuticals following the scandal.

The purpose of this paper is to theoretically and empirically analyze the impact of uncertainty concerning generic drug quality on generic drug use. The paper contributes to the existing literature in three ways; First, a new theoretical framework to analyze the physician's prescription decision, incorporating uncertainty concerning the quality of generic products, is presented. Second, given that changed approval procedures changes the physician's belief concerning generic drug quality, the theoretical model allows analysis of such effects upon the physicians prescription decision. Third, aggregation over physicians enables empirical analysis of the impact of uncertainty concerning generic drug quality on physician prescription behavior, using aggregated data.

In earlier studies of pharmaceuticals markets, the decision to switch from a brand name to a generic product has been modeled as a "now or never" decision made by the prescribing physician. In this paper the economic value of being able to defer the decision to switch to a later date, when more information about the quality and efficacy of the generic drug is available for the physicians, is studied. In order to do this the theories of real options, e.g., Dixit and Pindyck (1994), are used. ${ }^{3}$ There are three important characteristics of the problem which must be fulfilled for this approach to be appropriate. First, there must exist some degree of uncertainty about the

\footnotetext{
${ }^{2}$ In addition, Scott-Morton (1997) reports that other illegal activities were discovered during the investigations as well. Some generic firms had in fact been re-coating brand name products and submitting them as their own for the testing procedures. Needless to say, these products did very well in comparisons with the brand name product.

${ }^{3}$ Option pricing techniques have been used by Palmer and Smith (2000) to analyze the effects of uncertainty on economic evaluations of health care technologies in general. In their paper, they mention uncertainty about pharmaceutical prices and/or the introductions of new pharmaceuticals as examples where option pricing techniques might be used. In the present paper we concentrate on uncertainty concerning generic product quality instead.
} 
future "state of the world", i.e. some uncertainty about the quality and efficacy of the generic drug. Second, the decision to switch from a brand name drug to a generic product must entail some irreversible commitment of resources. Finally, the physician must have some discretion as to the timing of the switch from the brand name to the generic drug.

Along the lines of Hellerstein (1998), the prescribing physician is concidered to act as an agent for the consumer of the drug (generic or brand name). In addition, the physician is assumed to internalize a proportion of the patients utility because of direct pecuniary incentives to do so (e.g., the fear of loosing the patient to another physician), or because of altruistic considerations. As such, the physician must weigh costs and benefits when deciding which product to prescribe, brand name or generic. If the physician switches to a generic drug which is inefficient (or even harmful) this will reflect back on the physician. Since the drug is already consumed by the patient, the negative pharmacological effects on the patient are indeed irreversible, i.e. the cost of the decision to switch is in this sense irreversible.

The outline of this paper is as follows. In Section 2, the theoretical model is derived under the assumption that the physician acts as an agent for the consumer. In addition, Section 2 presents comparative statistics and implications for the utility maximization problem of the prescribing physician, as well as a method for aggregation across physicians. Section 3 presents empirical results incorporating uncertainty in the brand name versus generic prescription decision. Finally, Section 4 concludes the paper.

\section{The model}

In accordance with Hellerstein (1998) consider physician $j$ as an agent (for patient $i$ ) who prescribes the form of the drug (generic or brand name). Assume that patient $i$ is currently using the brand name drug. Define the physicians appropriately discounted expected utility, $U_{j t}$, and discounted expected disutility, $D_{j t}$, of changing dispensing habits from the brand name to the generic drug. ${ }^{4}$ The utility of the switch is a function of the utility gained

\footnotetext{
${ }^{4} U_{j t}$ is the expected discounted utility of switching to the generic drug at time $t$. The same applies for the disutility.
} 
by the patient, ${ }^{5}$

$$
U_{j t}=E_{t} \int_{t}^{\infty} f\left(\gamma_{j} u_{i t}\left(p_{\triangle}\right)\right) e^{-\rho \tau} d \tau,
$$

where $u_{i t}\left(p_{\triangle}\right)$ is patient $i$ :s utility of switching to the generic drug at time $t$. This utility is due to the positive difference between the price of the brand name drug, $p^{b r}$, and the price of the generic drug, $p^{g}$, i.e. $p_{\triangle}=p^{b r}-p^{g}>0$. The patients (and the physicians) utility is assumed increasing in the price differential, i.e. $\frac{\partial U}{\partial p_{\Delta}}>0$. The proportion of the patients utility/disutility that is internalized by the physician is given by $0 \leq \gamma_{j} \leq 1$. The physician may internalize a proportion of the patients utility/disutility because of direct pecuniary incentives to do so or because of altruistic considerations. The (subjective) discount rate is given by $\rho$. The disutility of switching to the generic drug is described by

$$
D_{j t}=E_{t} \int_{t}^{\infty} f\left(\gamma_{j} v_{i t}(q \triangle), c_{j}\right) e^{-\rho \tau} d \tau
$$

where $v_{i t}\left(q_{\triangle}\right)=\phi_{i t}$ is patient $i$ :s disutility of switching to the generic drug due to the expected positive difference in quality, $q_{\triangle}=q^{b r}-q^{g} \geq 0$. The patients (and the physicians) disutility is assumed increasing in the expected quality differential, i.e. $\frac{\partial D}{\partial q_{\triangle}}>0$. The physician is assumed to know the true quality of the brand name drug, $q^{b r}$, from prior experience (assumed for simplicity to be constant over time), as well as the current expected quality of the generic drug, $q^{g}$. Future values of $q^{g}$ is, however, not known due to uncertainty about the true quality of the generic drug. The uncertainty about future quality (or true quality) of the generic drug arises because the physician has no prior experience of the drug combined with less strict approval requirements for generic drugs. Finally, $c_{j}$ represents an exogenous switching cost, e.g., time spent searching for generic alternatives.

Assume that the physician has the ability to delay the decision of switching to the generic drug rather than making a prompt decision. This option would give the physician an opportunity to wait for more information about

\footnotetext{
${ }^{5}$ It is possible to include parameters reflecting wheather the patient has insurance covering the full, or part of the cost of the drug, and also parameters reflecting physicians taking social resposibility for health care costs. One might also include other direct costs or benefits associated with the decision. This will however not affect the main analysis in this paper.
} 
the true quality of the generic drug. The decision to switch is of course reversible, but the disutility absorbed given a switch to the generic drug, if the generic drug quality turn out bad, is assumed to be irreversible. ${ }^{6}$ Assume, for simplicity, that $U_{j t}$ is constant over time. The disutility absorbed by the patient from the expected lower generic quality is assumed to evolve according to the geometric Brownian motion

$$
d \phi_{i t}=\alpha \phi_{i t} d t+\sigma \phi_{i t} d z_{t}
$$

where $d z_{t}$ is the increment of a Wiener process, $\sigma$ the volatility coefficient and $\alpha$ the drift rate. The equation implies that the current expected disutility absorbed by the patient, $\phi_{i t}$, of the decision to switch to the generic drug is known to the physician. Future values are, however, uncertain due to the uncertainty concerning generic drug quality. The changes of the process are lognormally distributed with a variance growing linearly with the time horizon. Since the Brownian motion is a Markov process no other current or past information (past values of $\phi_{i t}$ ) affect the current value. Thus, $\phi_{i t+1}$ is affected only by $\phi_{i t}$. It is assumed that the physician's expectation about the disutility absorbed by the patient at time $t$ already contains (implicitly) all the relevant information from the past providing the physician with a necessary base for his/her decision. A negative drift rate, $\alpha<0$, is assumed, i.e. the patients disutility from using the generic drug, on average, decreases over time, to make the problem relevant. If the disutility where growing $(\alpha>0)$ the physician would never switch to the generic drug. Since the physicians disutility of switching to the generic drug is a proportion of the disutility absorbed by the patient this implies (assuming $\gamma_{j}$ constant over time) that $D_{j t}$ evolves according to the geometric Brownian motion,

$$
d D_{j t}=\alpha D_{j t} d t+\sigma D_{j t} d z_{t}
$$

The value of the physician's option to switch to the generic drug, at $t=0$, is denoted $F\left(D_{j}\right)$. The physician's objective is to choose the time to switch, $t=\tau_{j}$, that maximizes

$$
F\left(D_{j}\right)=\sup _{\tau_{j}} E_{0}\left[\int_{0}^{\tau_{j}}\left(U_{j t}-D_{j t}\right) e^{-\rho t} d t\right],
$$

\footnotetext{
${ }^{6}$ Irreversibility in the decision processes (or sunk costs) in combination with uncertainty about future costs or benefits, regarding a decision, creates an option value of waiting. See e.g., MacDonald and Siegel (1986) or Dixit and Pindyck (1994).
} 
subject to (3). The absolute value of the drift rate is assumed to be less than the discount rate, i.e. $|\alpha|<\rho$, since otherwise the solution of the optimal decision would be to wait until $D_{j t}=0$. The physician's decision rule will take the form of a critical value $D_{j}^{*}$ such that it is optimal to switch once $D_{j t} \leq D_{j}^{*}$. For values of $D_{j t}>D_{j}^{*}$ the optimal action will be to wait.

The Bellman equation ${ }^{7}$ for this optimal stopping problem in continuous time is given by

$$
F\left(D_{j t}\right)=\max \left\{\left(U_{j t}-D_{j t}\right),(1+\rho d t)^{-1} E\left[F\left(D_{j t}+d D_{j t}\right)\right]\right\},
$$

where $\left(U_{j t}-D_{j t}\right)$ is the value of an immediate switch. In the waiting region, $D_{j t}>D_{j}^{*}$, the second term is the larger of the two. Rewriting this term gives the equation governing the value of the option to switch,

$$
\rho F\left(D_{j t}\right) d t=E\left[d F\left(d D_{j t}\right)\right] .
$$

This equation states that over a time interval $d t$, the total expected gain of holding the option to switch, is equal to its expected rate of appreciation, due to improved knowledge about the generic drug quality. Using Ito's lemma $d F\left(d D_{j t}\right)$ can be expanded as

$$
d F\left(d D_{j t}\right)=F^{\prime}\left(D_{j t}\right) d D_{j t}+\frac{1}{2} F^{\prime \prime}\left(D_{j t}\right)\left(d D_{j t}\right)^{2}
$$

where $F^{\prime}\left(D_{j t}\right)=\frac{\partial F}{\partial D_{j t}}$ and $F^{\prime \prime}\left(D_{j t}\right)=\frac{\partial^{2} F}{\partial D_{j t}^{2}}$. Substituting (3) for $d D_{j t}$ and taking the expected value gives ${ }^{8}$

$$
E\left[d F\left(d D_{j t}\right)\right]=\alpha F^{\prime}\left(D_{j t}\right) D_{j t} d t+\frac{1}{2} \sigma^{2} D_{j t}^{2} F^{\prime \prime}\left(D_{j t}\right) d t .
$$

where the second term follows because the variance of $D_{j t}$ is of order $d t$. Substitution of (8) into (6) gives the Bellman equation (after dividing by $d t$ ) governing the value of the option to switch as

$$
\frac{1}{2} \sigma^{2} D_{j t}^{2} F^{\prime \prime}\left(D_{j t}\right)+\alpha F^{\prime}\left(D_{j t}\right) D_{j t}-\rho F\left(D_{j t}\right)=0 .
$$

\footnotetext{
${ }^{7}$ Assuming an infinite time horizon for the decision problem simplifies the analysis, since this implies independence from time $t$ as such. This means that the calender time by itself has no effect. The Bellman equation or value function is then common for all periods, although it will be evaluated at different points $D_{j t}$.

${ }^{8}$ Note that $E(d z)=0$ and that terms of higher order than $d t$ vanishes in the limit. We assume an infinite time horizon and that $\alpha$ and $\sigma$ is independent of time. By assuming this the problem is simplified resulting in an ordinary differential equation instead of a stochastic.
} 
The value of the option to switch to the generic drug satisfies this equation along with boundary conditions

$$
\begin{aligned}
F(0) & =U_{j} \\
\lim _{D_{j t \rightarrow \infty}} F\left(D_{j t}\right) & =0 \\
F\left(D_{j}^{*}\right) & =U_{j}-D_{j}^{*} \\
F^{\prime}\left(D_{j}^{*}\right) & =-1
\end{aligned}
$$

The first boundary condition describes that zero is an absorbing state for the geometric Brownian motion, i.e. if the disutility of changing to the generic drug becomes zero it will stay at zero, and the value of the option to switch is simply $U_{j}$. The second condition describes that when the disutility grows enough, the probability of reaching $D_{j}^{*}$ goes to zero, and the value of the switch option goes to zero. The third condition is the "value-matching condition" expressing the fact that at the optimal trigger level of disutility the pay-off is simply the net utility pay-off. The fourth condition is the "smooth pasting condition", e.g., Dixit (1993).

The general solution to equation (9) is given by

$$
F\left(D_{j}\right)=A_{1} D_{j}^{\beta_{1}}+A_{2} D_{j}^{\beta_{2}}
$$

where $\beta_{1}$ and $\beta_{2}$ are the positive and the negative root, respectively, of the fundamental equation

$$
Q=\frac{1}{2} \sigma^{2} \beta(\beta-1)+\alpha \beta-\rho=0 .
$$

The two roots are given by

$$
\beta_{i}=\frac{1}{2}-\alpha / \sigma^{2} \pm \sqrt{\left[\alpha / \sigma^{2}-\frac{1}{2}\right]^{2}+2 \rho / \sigma^{2}}, i=1,2 .
$$

The second boundary condition implies that $A_{1}=0$ and gives the solution

$$
F\left(D_{j}\right)=A_{2} D_{j}^{\beta_{2}},
$$

where $A_{2}$ and $D_{j}^{*}$ are still to be determined. Utilizing the value-matching and smooth pasting condition gives

$$
A_{2}=\frac{\left(\beta_{2}-1\right)^{\left(\beta_{2}-1\right)}}{\beta_{2}^{\beta_{2}} U_{j}^{\left(\beta_{2}-1\right)}}
$$


and

$$
D_{j}^{*}=\frac{\beta_{2}}{\left(\beta_{2}-1\right)} U_{j}
$$

The critical value $D_{j}^{*}$, at which it is optimal to switch to the generic drug, is a fraction of the utility gained by the switch since $0<\frac{\beta_{2}}{\left(\beta_{2}-1\right)}<1$. Note that $\beta_{2}$ is the negative root of the fundamental equation. The implication of this is that uncertainty of the generic drug quality creates a value of waiting for more information. For comparison the optimal behavior without uncertainty would be to switch when $D_{j t} \leq U_{j t}$.

The optimal time to switch (e.g., Oksendal, 1995) is given by $\tau_{j}^{*}$, where

$$
\tau_{j}^{*}=\inf \left\{t>0: D_{j t} \notin\left(\infty, D_{j}^{*}\right)\right\} .
$$

Hence, $\tau_{j}^{*}$ is the first time at which the physicians disutility exit the interval $\left(\infty, D_{j}^{*}\right)$. If $D_{j 0} \leq D_{j}^{*}$, then $E\left(\tau_{j}^{*}\right)=0$, and an immediate switch to the generic drug is the optimal behavior. Now instead assume that $D_{j 0} \in\left(\infty, D_{j}^{*}\right)$, i.e. the option to wait has a positive value. Using Ito's Lemma (e.g., Ito and Mckean, 1965) on equation (3), one can show that the value of the physicians disutility of switching to the generic drug at time $t \geq 0$, starting at $D_{j 0}$, is given by $D_{j t}=D_{j 0} \exp \left(\alpha-1 / 2 \sigma^{2}\right) t+\sigma z_{t}$. From this the expected time to switch, assuming $\alpha-1 / 2 \sigma^{2}<0$, for a physician is given by

$$
E\left(\tau_{j}^{*}\right)=\ln \left[\frac{D_{j}^{*}}{D_{j 0}}\right] /\left[\alpha-\frac{1}{2} \sigma^{2}\right],
$$

where $D_{j}^{*} \leq D_{j 0}$.

\subsection{Comparative statistics and implications}

One of the main objectives of this paper is to study how the physician's optimal prescription behavior is affected by changes in uncertainty concerning the quality of the generic drug. In the Appendix it is shown that

$$
\frac{\partial D_{j}^{*}}{\partial \sigma}<0
$$

and, further, that

$$
\frac{\partial E\left(\tau_{j}^{*}\right)}{\partial \sigma}=\frac{D_{j}^{*}(\cdot)\left[\alpha-\frac{1}{2} \sigma^{2}\right] / D_{j}^{*}+\left[\ln D_{j}^{*}-\ln D_{j 0}\right] \sigma}{\left[\alpha-\frac{1}{2} \sigma^{2}\right]^{2}}>0
$$


This means that increased uncertainty about future disutility of switching to the generic drug will decrease the optimal critical switching value and, hence, increase the expected time until switching. ${ }^{9}$

If physicians believe generic drugs to be less reliable than brand name drugs (as reported by Fridman et al 1987), changes in approval procedures for generic drugs might have unintended consequences. If changes in approval procedures increase uncertainty about the quality of generic drugs, this will make physicians more reluctant (i.e. wait longer) to prescribe generic drugs. This negative effect reduces the intended positive effects on generic entry and generic competition of changed approval procedures. As such, the total effects of changed approval procedures on generic entry and generic competition is a question for empirical research. ${ }^{10}$

\section{$2.2 \quad$ Aggregating across physicians}

The theoretical model presented above applies to the individual prescribing physicians, whereas available data only makes it possible to study behavior at an aggregated level. As such, aggregation across physicians has to be considered. Given that a physician prescribed the brand name drug prior to time $t$, he/she will switch to the generic drug at time $t$ if

$$
\frac{\beta_{2}}{\left(\beta_{2}-1\right)} U_{j t}-D_{j t}^{*} \geq 0
$$

Assuming heterogeneity concerning physicians level of internalization, $\left(\gamma_{j}\right)$, of the patients disutility of using the generic drug, each physician will face a unique decision problem subject to their own individual specific motions of disutility, $D_{j}$. This implies that comparing the optimal switching levels, $D^{*}$

\footnotetext{
${ }^{9}$ Note that this corresponds rather well to what happened in the US market after the generic drug scandal, according to Gupta (1996). He found that increased uncertainty about generic drug quality made pharmacists use more brand name drugs after the scandal, at least for personal use.

${ }^{10}$ Grabowski and Vernon $(1992,1996)$ have studied the effects of the 1984 WaxmanHatch act. The act was designed to promote price competition by introducing an abbreviated new drug application process for generic drugs, while restoring some of the effective patent time for brand name drugs. They report that after the 1984 act, the average generic market share has increased in the US pharmaceuticals market, indicating that the price effect might have outweighed the effect of increased uncertainty concerning the quality of generic drugs.
} 
for two physicians may not reveal who will switch to the generic drug first. ${ }^{11}$ To be able to relate the decision described in equation (17) to the change of market share of the brand name drug, the attention is instead turned to the expected time to switch.

Assume that expected switching times, $\tau$, among physicians who has not prior switched to the generic drug, have, at time $t$, a distribution given by $\int_{t}^{\infty} g(\tau) d \tau$. Assume this distribution to be the same for all periods and independent of the market share of the brand name drug. The expected share of physicians switching to the generic drug during the discrete time period $t$ to $t+1$ is then given by $\int_{t}^{t+1} g(\tau) d \tau$. Formally, the change of market share of the brand name drug may now be related to the share of physicians switching to the generic drug as

$$
\frac{s_{t+1}-s_{t}}{s_{t}}=\frac{\int_{t}^{t+1} g(\tau) d \tau}{\int_{t}^{\infty} g(\tau) d \tau}=h\left(p_{\triangle}, q_{\triangle}, c, \sigma, \alpha\right)
$$

where $s_{t}$ is the share of physicians patronizing the brand name drug at time $t$. Equation (22) gives the relative change of market share for the brand name drug as a function of the parameters affecting the distribution of switching times. All variables affecting the utility of the physician as presented by equation (1), and the disutility as presented by equation (2), will enter the analysis by affecting the distribution of $\tau^{*}$ through $D$ and $D^{*}=U \beta_{2} /\left(\beta_{2}-1\right)$. Note that increased uncertainty about generic drug quality affects equation (22) by shifting the mean of the switching time distribution to the right. Fewer prescribing physicians will then reach their critical value during $(t, t+1)$ and switch from brand name to generic substitutes. This means that brand name loss of market share will be slower for drugs where uncertainty is large and/or increases. Variables increasing the utility of switching to a generic product will make the loss of brand name market share more dramatic, while the opposite is true for variables who increases the disutility of the switch.

\footnotetext{
${ }^{11} \mathrm{~A}$ lower $D^{*}$ may not imply a longer waiting period before switching, since the starting value $D_{j 0}$ is unique for each physician (due to different $\gamma_{j}$ among physicians). Hence, an aggregation approach building on distributional assumptions of $D_{j}^{*}$, see Aronsson et al (2001), is inappropriate in the current setting.
} 


\section{Empirical test}

Physicians perceptions of the quality of generic drugs are obviously not easily observable and an uncertainty measure is thus difficult to construct. The available data do not contain any measure concerning changes in uncertainty of generic drug quality over time. To test whether uncertainty affects physician prescription behavior, the strategy is therefore to compare pharmaceuticals with different levels of uncertainty concerning generic quality. Assuming that a higher level of uncertainty mainly affects the generic versions of the drug (since the prescribing physician has prior experience with the brand name version), brand name drugs for substances with a high level of uncertainty should lose market share to generics more slowly. As such, the empirical test procedure will use a substance related measure of the uncertainty concerning generic drug quality, $\sigma$, while trying to control for all other factors (i.e. $\left.p_{\triangle}, q_{\triangle}, c, \alpha\right)$ affecting physician prescription behavior.

\subsection{Data}

Quarterly time-series data of prices and quantities for each brand name product and its generic substitutes from 1972 to 1996 are used in this study. These data have been provided by the Swedish Medical Product Agency (SMPA) and cover 5 of 14 different fields of use, as defined by the SMPA. The data refers to twelve different substances, all of which have a minimum sale of ten thousand packages each quarter for the chosen package size. ${ }^{12}$ The price of generic products used in the estimations is the average price of generic substitutes in each submarket, measured as a quantity weighted average. Table 1 reports the average market share (measured in quantity) as well as means and standard deviations for the relative prices (i.e. the price of the brand name product relative to the average price of the generic substitutes) for each original substance and its generic substitutes during the considered period.

\footnotetext{
${ }^{12}$ The products used in the study refer to the dose and package size with the largest registered sales.
} 
Table 1: Market shares and relative prices.

\begin{tabular}{lccr}
\hline \hline Substance & $\begin{array}{l}\text { M-share } \\
\text { Brand }\end{array}$ & $\begin{array}{c}\text { M-share } \\
\text { Generic }\end{array}$ & $\begin{array}{r}\text { Relative price } \\
\text { (std. dev) }\end{array}$ \\
\hline Cimetedine & $64.57 \%$ & $35.43 \%$ & $1.62(0.66)$ \\
Furosemide & $47.40 \%$ & $52.60 \%$ & $1.57(0.45)$ \\
Atenolol & $85.51 \%$ & $14.49 \%$ & $1.58(0.44)$ \\
Pindolol & $97.63 \%$ & $2.37 \%$ & $1.36(0.18)$ \\
Propranolol & $96.98 \%$ & $3.02 \%$ & $1.87(0.55)$ \\
Indomethacine & $63.56 \%$ & $36.44 \%$ & $1.15(0.10)$ \\
Naproxen & $44.30 \%$ & $55.70 \%$ & $1.44(0.37)$ \\
Allopurinol & $93.03 \%$ & $6.97 \%$ & $1.33(0.22)$ \\
Paracetamol/ & & & \\
Codeine & $92.85 \%$ & $7.15 \%$ & $1.23(0.10)$ \\
Diazepam & $41.68 \%$ & $58.32 \%$ & $1.17(0.12)$ \\
Clomipramine & $39.53 \%$ & $60.47 \%$ & $1.44(0.19)$ \\
Timolol & $82.08 \%$ & $17.92 \%$ & $1.75(0.40)$ \\
\hline
\end{tabular}

Note: The figures in the table refer to average market shares and relative prices $\left(\mathrm{p}^{b r} / \mathrm{p}^{g}\right)$ during the estimation period.

\subsection{The regression model}

The empirical model is specified as

$$
\begin{aligned}
\frac{s_{i t}-s_{i t-1}}{s_{i t-1}}= & \alpha_{j}+\alpha_{i} T+\beta_{1}\left(p_{i t}^{b r} / p_{i t}^{g}\right)+\beta_{2} G E N_{i t}+\beta_{3} D R E F \\
& +\beta_{4} A D S_{i t}+\beta_{5} U N C+u_{i t}
\end{aligned}
$$

where $u_{i t}$ is a random term, assumed to be i.i.d. across substances, and where $\alpha_{j}$ are fix effects. These fixed effects represents the five fields of use, $j=1, \ldots, 5$, (as defined by the SMPA) and are assumed to capture differences in treatment times, the severity of the underlying disease etc. The term $T$ represents a time trend to capture the trend in the stochastic quality process described in equation (3). $p_{i t}^{b r} / p_{i t}^{g}$ represents the relative price difference between the brand name drug and the generic version, and is defined as the price of the brand name drug, $p^{b r}$, over the average price of the generic substitutes, $p^{g}$. The number of generic versions of the pharmaceutical substance in question is measured by the variable $G E N_{i t}$, assumed to capture changes 
and differences in the switching cost, $c$, while $D R E F$ is a dummy variable representing the introduction of the Swedish reference price system in 1993. The system limits the reimbursement to patients who purchase pharmaceuticals to 110 percent of the price of the least-cost identical generic alternative. Before 1993, the patient had to pay all cost below 120 SEK themselves, while costs exceeding 120 SEK were borne by the National Social Insurance Board (NSIB). After paying a total of 1500 SEK, all costs were reimbursed by the NSIB. In addition, the policies for registration of generic products were changed in 1993 in order to make it easier for generic manufacturers to enter the market. The dummy variable DREF thus capture a net effect on brand name market share caused by increased uncertainty, $\sigma$, and of decreased switching costs, $c$, caused by the introduction of the new reimbursement and approval procedures. However, as this variable measures a net effect of the new reimbursement system and changed approval procedures, it cannot be used in order to test the theoretical model presented above.

$A D S_{i t}$ represents the number of ads in Läkartidningen ${ }^{13}$ for the brand name pharmaceutical in relation to the total number of ads for that pharmaceutical substance, brand name and generic, and is used as a proxy in order to control for differences in quality (i.e. $q_{\triangle}$ ) between brand name drugs and generics. This follows from the signaling model developed by Spence (1973). High quality producers of a pharmaceutical product uses advertising as an instrument to signal high quality, if their cost of using this instrument is lower than for the low quality producer. As pharmaceuticals are experience goods, producers will not receive repeat purchases if quality is low, making the cost of using advertising as a signaling device high for low quality producers. (see e.g., Tirole, $1988 \mathrm{p} \mathrm{119).}{ }^{14}$

Finally, $U N C$ measures uncertainty about the quality of the generic drug. This is measured in four different ways, relating to models one to four below. The three first measures are dummy variables, while the fourth is a continuous variable. The first two measures, $U N C_{1}$ and $U N C_{2}$, are related to those pharmaceutical substances who have been reported for side-effects during the last six years and who are made public in the SMPA's compilation

\footnotetext{
${ }^{13}$ Läkartidningen is a weekly medical journal directed towards physicians in Sweden. This is the largest official journal where prescription pharmaceuticals are advertised in the Swedish market, reaching approximately 90 percent of the prescribing physicians.

${ }^{14}$ It should be noted that the number of ads might affect the switching cost, $c$, in the theoretical model as well. As such, the number of ads is likely to measure the net effect of advertising on quality differences and switching costs.
} 
and assessment of side-effects reported from the healthcare sector. ${ }^{15}$ The first measure, $U N C_{1}$, represents those substances in the sample which have been reported to the SMPA for having side-effects $(1=$ side effects). The second measure, $U N C_{2}$, also includes substances which have been reported by the SMPA for side-effects when used in combination with other pharmaceutical products. A third measure, $U N C_{3}$, includes all pharmaceutical substances which have the same four figure ATC-code ${ }^{16}$ as those pharmaceuticals which are included in our second measure, $U N C_{2}$. Finally, a fourth uncertainty measure is constructed by counting the reported number of rare side-effects in FASS ${ }^{17}$ in relation to the total number of side effects.

\subsection{Empirical results}

Four different versions of equation (22), relating to the different uncertainty measures, $U N C_{1}$ to $U N C_{4}$, are estimated. Comparison of the results for estimators accounting for the unbalanced panel structure with estimators not accounting for this, indicate no qualitative differences. ${ }^{18}$ The initial estimations indicate a significant autocorrelation in the residuals at lag 2, for all specifications. Since robust (heteroskedasticity and autocorrelation) covariance estimators for the unbalanced case are not readily availably ${ }^{19}$, and since the effect of the unbalanced panels are small, estimation techniques not accounting for the unbalanced panel structure were used. A Newey-West (1987) covariance estimator (lag 2) has been utilized. The results from the regressions are reported in Table $2 .^{20}$

\footnotetext{
${ }^{15}$ Two substances, Allopurinol and Propranolol, have been reported for side effects, while Paracetamol have been reported for side effects in combination with other pharmaceuticals. These reports (in Swedish) can be found on the SMPA website at the following address: http://www.mpa.se/biverkningar/biv02/bivmain02.shtml

${ }^{16}$ The Anatomical Therapeutic Chemical (ATC) classification system divides drugs into different groups according to the organ or system on which they act and their chemical, pharmacological and therapeutic properties.

${ }^{17}$ FASS is a physicians guide to pharmaceutical products sold in the Swedish pharmaceuticals market.

${ }^{18}$ The models were estimated with least squares techniques utilizing the LIMDEP software package.

${ }^{19} \mathrm{~A}$ least squares estimator accounting for the unbalanced panel structure was estimated for models including an AR(1) error structure. The estimation results were similar to those reported in Table 2 .

${ }^{20}$ The fixed effects have been left out in order to save space.
} 
Table 2: Estimation results. (Standard errors in paranthesis)

\begin{tabular}{lllll}
\hline \hline Variable & $U N C_{1}$ & $U N C_{2}$ & $U N C_{3}$ & $U N C_{4}$ \\
\hline$T$ & $0.675 \times 10^{-4 *}$ & $0.102 \times 10^{-3}$ & $0.262 \times 10^{-3}$ & $0.628 \times 10^{-5}$ \\
& $\left(0.129 \times 10^{-3}\right)$ & $\left(0.144 \times 10^{-3}\right)$ & $\left(0.182 \times 10^{-3}\right)$ & $\left(0.118 \times 10^{-3}\right)$ \\
$p_{i t}^{o} / p_{i t}^{g}$ & $-0.032^{*}$ & $-0.033^{*}$ & $-0.031^{*}$ & $-0.027^{*}$ \\
& $(0.012)$ & $(0.013)$ & $(0.012)$ & $(0.011)$ \\
$G E N_{i t}$ & -0.003 & -0.002 & -0.003 & -0.002 \\
& $(0.002)$ & $(0.002)$ & $(0.002)$ & $(0.002)$ \\
$D R E F$ & -0.011 & -0.013 & -0.016 & -0.009 \\
& $(0.014)$ & $(0.014)$ & $(0.015)$ & $(0.012)$ \\
$A D S_{i t}$ & $-0.14 \times 10^{-4}$ & $-0.810 \times 10^{-5}$ & $0.345 \times 10^{-4}$ & $-0.272 \times 10^{-4}$ \\
& $\left(0.279 \times 10^{-4}\right)$ & $\left(0.297 \times 10^{-4}\right)$ & $\left(0.342 \times 10^{-4}\right)$ & $\left(0.309 \times 10^{-4}\right)$ \\
$U N C$ & $0.023^{*}$ & $0.023^{*}$ & $0.030^{*}$ & 0.011 \\
& $(0.005)$ & $(0.008)$ & $(0.009)$ & $(0.013)$ \\
\hline
\end{tabular}

*Significant at the 5 percent level.

Based on theory, the following results are expected when estimating the above model. First, the relative price difference is expected to have a negative effect on the loss of brand name market share, i.e. if the brand name product is priced high relative to the generics, the utility gained by switching will be large and physicians will substitute away from the brand name product. Second, the brand name product will lose market shares faster if more generic versions enter the market, i.e. the parameter estimate of $\beta_{2}$ is expected to be negative. The motivation for this is that the switching cost included in the disutility, as presented by equation (3), will decrease in the number of generics, i.e. less time has to be spent searching for a generic alternative. Third, if the brand name product is heavily advertised compared to the generic versions of the product, this is assumed to be a signal that the brand name product is of high quality, and the brand name product is expected to lose market share more slowly. ${ }^{21}$ Fourth, the introduction of the Swedish reference price system is assumed to lower the switching costs, while increasing the uncertainty concerning generic drug quality. Thus, the sign of the parameter $\beta_{3}$ is ambigous. Fifth, as the number of ads in Läkartidningen is used as a proxy for quality differences, where heavily advertised pharmaceuticals are assumed to be of high quality, the parameter $\beta_{4}$ is assumed to be negative. Finally, the parameter estimate for $\beta_{5}$ is expected to be positive if

\footnotetext{
${ }^{21}$ Note that this will also be the case if the advertising of brand name products increase the switching cost of the prescribing physicians.
} 
uncertainty about the quality of generic drugs are higher for those substances included in our uncertainty measures. This means that the physicians will have a higher value of waiting for more information concerning the quality of generic products for these substances. This should especially be true for those pharmaceuticals which have been reported to the SMPA for having side-effects. The "wait-and-see" strategy will lead to a slower loss of market share for the brand name product for these substances.

The relative price parameter is negative and statistically significant at the five percent level in all four models. The estimates concerning the number of generic versions and the introduction of the reference price system are negative as expected, but not statistically significant. In addition, advertising intensity of the brand name product does not seem to affect the loss of market share for such products in any significant way.

The estimates for the uncertainty parameter is positive in all four models. In the first three models, where different dummy variable constructions are used to measure uncertainty, the estimates are significant at the five percent level. This indicates that uncertainty concerning generic drug quality affect the prescribing physicians decision, whether to switch to the generic drug or not, according to the theory.

\section{Conclusion}

In earlier studies of pharmaceuticals markets, the decision to switch from a brand name to a generic product has been modeled as a "now or never" decision made by the prescribing physician. In this paper, the economic value for the physicians of being able to defer the decision to switch to a later date, when more information about the quality of the generic drug is available, is studied. This is accomplished by means of a real options theoretical approach, in which it is shown that uncertainty about generic drug quality gives the physician a value of waiting before switching to the generic drug. The physicians optimal behavior is thus to wait longer before prescribing the generic drug, compared to a situation where generic drug quality is known with certainty. This theoretical prediction coincides with the findings of Gupta (1996), following the generic drug scandal in the US in 1989. The scandal affected the physicians and pharmacists confidence in generic drug quality and the pharmacists included in the study by Gupta reported to have increased their use of brand name drugs, at least for personal use, 
following the scandal. In addition, the small empirical study on Swedish data supports the theoretical findings in this paper and indicate that uncertainty about the quality of generic drugs do affect physician prescription behavior.

The theoretical approach allows analysis of the effects of changed approval procedures on generic drug use as well. Reducing the approval requirements for generic drugs to enhance competition, as has been done in several countries, could at the same time increase uncertainty about the generic drug quality. If this is the case, our model predicts that these changes will actually discourage physicians from prescribing generic drugs, ceteris paribus. The studies of the introduction of the Waxman-Hatch act in 1984 by Grabowski and Vernon $(1992,1996)$ indicates that the price effect outweighed the effects of increased uncertainty concerning generic drug quality on physician prescription behavior. However, this study suggests that the effects of the act could have been larger, if policies also had been aimed at minimizing uncertainty concerning generic drug quality. 


\section{Appendix}

First it is shown that

$$
\frac{\partial D_{j}^{*}}{\partial \sigma}<0
$$

The wedge between $D_{j}^{*}$ and $U_{j}$ is depending on the negative root, $\beta_{2}$, of the fundamental equation

$$
Q=\frac{1}{2} \sigma^{2} \beta(\beta-1)+\alpha \beta-\rho=0 .
$$

To understand how the critical value is affected by uncertainty totally differentiate this equation and evaluate the derivatives at $\beta_{2}$,

$$
\frac{\partial Q}{\partial \beta} \frac{\partial \beta_{2}}{\partial \sigma}+\frac{\partial Q}{\partial \sigma}=0
$$

The derivatives are given by

$$
\frac{\partial Q}{\partial \beta}=\left(\beta-\frac{1}{2}\right) \sigma^{2}-\alpha<0
$$

and

$$
\frac{\partial Q}{\partial \sigma}=\sigma \beta(\beta-1)>0
$$

where $\beta_{2}<0, \sigma \geq 0$ and $\alpha \leq 0$. This implies that $\frac{\partial \beta_{2}}{\partial \sigma}>0$, (i.e. becomes less negative) and thus that $\frac{\beta_{2}}{\left(\beta_{2}-1\right)}$ decreases.

The second proposition is given by

$$
\frac{\partial E\left(\tau_{j}^{*}\right)}{\partial \sigma}=\frac{D_{j}^{*}(\sigma)\left[\alpha-\frac{1}{2} \sigma^{2}\right] / D_{j}^{*}+\left[\ln D_{j}^{*}-\ln D_{j 0}\right] \sigma}{\left[\alpha-\frac{1}{2} \sigma^{2}\right]^{2}}>0
$$

where $D_{j}^{*}(\sigma)=\frac{\partial D_{j}^{*}(\sigma)}{\partial \sigma}$. This inequality holds if

$$
D_{j}^{*}(\sigma)\left[\alpha-\frac{1}{2} \sigma^{2}\right] / D_{j}^{*}>\ln \left[D_{j}^{*} / D_{j 0}\right] \sigma,
$$

since $\alpha-\frac{1}{2} \sigma^{2}<0, D_{j}^{*} \leq D_{j 0}$ and $D_{j}^{*}(\sigma)=\partial D_{j}^{*} / \partial \sigma<0$. Rewriting and taking the exponential on both sides gives

$$
\exp \left[\frac{D_{j}^{*}(\sigma)\left[\alpha-\frac{1}{2} \sigma^{2}\right]}{D_{j}^{*} \sigma}\right]>\frac{D_{j}^{*}}{D_{j 0}} .
$$

Since $\exp (x>0)>1$ and $D_{j}^{*} \leq D_{j 0}$ the inequality must hold. 


\section{References}

Andersson, F. and P. Hertzman, 1993. Effective Patent Life of Drugs in Sweden - A Comparison with International Studies. Managerial and Decision Economics, 14, 53-63.

Aronsson, T., M.A. Bergman and N. Rudholm, 2001. The Impact of Generic Competition on Brand Name Market Shares - Evidence from Micro Data, Review of Industrial Organization, 19, 423-433.

Dixit, A., 1993. The Art of Smooth Pasting. Harwood Academic Publishers.

Dixit, A. and R. Pindyck, 1994. Investment Under Uncertainty. Princeton University Press, Princeton.

Fridman, D., A. Jaffe and S. Steinhardt, 1987. Physicians' Attitudes Toward and Knowledge About Generic Drug Substitution, New York State Journal of Medicine, 87, 539-542.

Grabowski, H.G. and J.M. Vernon, 1992. Brand Loyalty, Entry and Price Competition in Pharmaceuticals After the 1984 Drug Act, Journal of Law and Economics, 35, 331-350.

Grabowski, H.G. and J.M. Vernon, 1996. Longer Patents for Increased Generic Competition in the US, PharmacoEconomics, 10, 110-123.

Gupta, P.B., 1996. Survey of Pharmacists: Impact of the Generic Drug Scandal and Implications for Marketing Generic Drugs, Health Marketing Quarterly, 13, 109-120.

Hartley, K., R.J. Lavers and A.K. Maynard,1986. Regulation and Development Times in the UK Pharmaceutical Industry, Scottish Journal of Political Economy, 33, 355-369.

Hellerstein, J.K., 1998. The Importance of the Pharmacist in the Generic versus Brand-name Prescription Decision. RAND Journal of Economics, 29, 108-136.

Ito, K. and H.P. Mckean, 1965. Diffusion Processes and Their Sample Paths. Springer-Verlag, Berlin.

McDonald, R. and D. Siegel, 1986. The Value of Waiting to Invest. Quarterly Journal of Economics, 101, 707-728.

Newey, W. and K. West, 1987 A simple, positive semi-definite, heteroskedasticity and autocorrelation consistent covariance matrix, Econometrica, 55, 703-708.

Oksendal, B., 1995. Stochastic Differential Equations: An Introduction with Applications. Fourth edition, Springer-Verlag, Berlin. 
Spence, M., 1973. Job Market Signaling, Quarterly Journal of Economics, 87, 355-374.

Scott-Morton, F.M., 1997. The Objectives of the FDA'S Office of Generic Drugs, NBER Working Paper 6143.

Scott Morton, F.M., 1999. Entry Decisions in the Generic Pharmaceutical Industry, Rand Journal of Economics, 30, 421-440.

Tirole, J., 1988. The Theory of Industrial Organization, MIT Press, Cambridge, Massachusetts. 